\title{
INFLUÊNCIA DE UM PROGRAMA DE ATIVIDADE FÍSICA DE LONGA DURAÇÃO SOBRE A FORÇA MUSCULAR MANUAL E A FLEXIBILIDADE CORPORAL DE MULHERES IDOSAS
}

\author{
Rebelatto JR ${ }^{1}$, Calvo JI ${ }^{2}$, Orejuela JR ${ }^{2}$, Portillo JC ${ }^{3}$ \\ ${ }^{1}$ Departamento de Fisioterapia, Universidade Federal de São Carlos, São Carlos, SP \\ ${ }^{2}$ Esc. Universitária, área de Fisioterapia, Universidade de Salamanca, Salamanca, Espanha. \\ ${ }^{3}$ Departamento de Reabilitação, Escola de Medicina, Universidade de Madri, Madri, Espanha. \\ Correspondência para: José Rubens Rebelatto, Departamento de Fisioterapia, Universidade Federal de São Carlos, \\ Rod. Washington Luiz Km 235, CEP 13565-905, São Carlos ,SP, e-mail: rubens@power.ufscar.br.
}

Recebido: 28/06/2005 - Aceito: 10/08/2005

\begin{abstract}
RESUMO
Objetivo: O presente trabalho examinou a influência de um programa de exercícios físicos prolongados (dois anos) sobre a força muscular e a flexibilidade corporal de mulheres idosas (60-80 anos), não institucionalizadas, da Província de Salamanca (Espanha). Método: Participaram 32 sujeitos, escolhidos dentre os participantes do Programa de Revitalização Geriátrica desenvolvido pela Universidade de Salamanca. Os critérios para escolha dos sujeitos foram: serem do sexo feminino, terem entre 60 e 80 anos e terem participado de pelo menos $74 \%$ do programa de atividades físicas. Foram realizadas 174 sessões de atividade física, durante 58 semanas, com freqüência de três vezes por semana e duração de 50 a 55 minutos cada uma. No período do experimento foram realizadas quatro medidas da força muscular manual, por meio de manômetro de pressão, e da flexibilidade corporal, por meio do teste sit and reach. Os dados foram analisados por meio de ANOVA com medidas repetidas. Resultados: Tanto em relação à força muscular, quanto à flexibilidade os dados apontam para a ausência de diferenças significativas $(p=0,005)$. Conclusões: O programa contribuiu para a manutenção da força de preensão manual no período de dois anos e, em relação à flexibilidade corporal, os dados indicam a necessidade de reprogramação dos exercícios destinados ao desenvolvimento de tal capacidade.
\end{abstract}

Palavras-chave: atividade física, idosos, força muscular, flexibilidade corporal.

\section{ABSTRACT \\ Influence of a Long-Term Physical Activity Program on Hand Muscle Strength and Body Flexibility Among Elderly Women}

Objective: The present study evaluated the influence of a two-year physical activity program on muscle strength and body flexibility among non-institutionalized elderly women (60-80 years old), from Salamanca Province in Spain. Method: Thirty-two individuals were chosen to take part in the Geriatric Revitalization Program at Salamanca University. The selection criteria were that they should be female, between 60 and 80 years old, with an attendance rate of at least $74 \%$ in the physical activity program. There were 174 physical activity sessions, over a 58-week period, three times a week, lasting 50-55 minutes each. During this period, four measurements of hand muscle strength were made, using a pressure manometer, and also of body flexibility, using the sit and reach test. The data were analyzed using ANOVA with repeated measurements. Results: There were no significant differences in relation to either muscle strength or flexibility ( $\mathrm{p}=0.005)$. Conclusions: The program contributed towards maintaining hand grip strength over the two-year period. For body flexibility, the data indicate the need to reprogram the exercises aimed at developing this capacity.

Key words: physical activity, elderly people, muscle strength, body flexibility. 


\section{INTRODUÇÃO}

O processo de envelhecimento do ser humano tem sido um foco de atenção crescente por parte de cientistas em todo o mundo, na medida em que a quantidade de indivíduos que chega à chamada "terceira idade" aumenta e, por decorrência, faz com que tanto os problemas de saúde característicos desse período da vida quanto os vários aspectos relativos à qualidade de vida dessa população sejam objetos de preocupação e de estudos.

Segundo Gonçalves ${ }^{1}$, em 2025, 15\% da população brasileira (34 milhões) estará acima de 60 anos, sendo que nesse período haverá um aumento médio de 6,5\% de idosos ao ano e, ao mesmo tempo, uma redução nos números absolutos de jovens entre 0 e 14 anos. Esse mesmo autor relata que "com o envelhecimento da sociedade, o brasileiro vai conviver mais com idosos, permitindo às gerações que amadurecerem ter um paradigma do que é ser velho. Para combater a inércia à qual os idosos estão confinados, nada melhor do que o movimento. É uma forma de adiar o repouso absoluto".

O envelhecimento biológico é um fenômeno multifatorial que está associado a profundas mudanças na atividade das células, tecidos e órgãos, como também com a redução da eficácia de um conjunto de processos fisiológicos. Do ponto de vista funcional, a população de indivíduos chamados "da terceira idade"- cuja expectativa de vida tem aumentado significativamente nos últimos anos - caracteriza-se, entre outros aspectos, por um decréscimo do sistema neuromuscular, verificando-se a perda de massa muscular, debilidade do sistema muscular, redução da flexibilidade, da força, da resistência e da mobilidade articular, fatores que, por decorrência, determinam limitação da capacidade de coordenação e de controle do equilíbrio corporal estático e dinâmico². No que se refere à força muscular e, especificamente à força de preensão das mãos, Fleck e Kraemer ${ }^{3}$ descreveram uma regressão de $3 \%$ ao ano em homens e $5 \%$ ao ano em mulheres, após um estudo longitudinal com duração de quatro anos. Em relação à flexibilidade, a "elasticidade” dos tendões, ligamentos e cápsulas articulares diminuem com a idade devido à deficiência de colágeno, determinando que durante a vida ativa, adultos percam algo como $8-10 \mathrm{~cm}$ de flexibilidade na região lombar e no quadril, quando medido por meio do teste sit and reach ${ }^{4}$. A restrição na amplitude de movimento das grandes articulações torna-se maior no período da aposentadoria e, eventualmente, a independência é ameaçada porque o indivíduo não consegue utilizar um carro ou um banheiro sem adaptações, subir uma pequena escada ou mesmo combinar os movimentos de vestir-se e pentear os cabelos.

Além dos aspectos citados, verificam-se também nesses indivíduos modificações na composição corporal, diminuição na quantidade de peso, na altura, na densidade mineral óssea, nas necessidades energéticas e no metabolismo, devido à vida sedentária e à diminuição da massa muscular ${ }^{5}$. O próprio sedentarismo associado à menor ingestão alimentar e a outras mudanças ligadas ao envelhecimento, tais como menor motilidade e absorção intestinal, alteração do metabolismo de glicídios, cálcio, ferro, micronutrientes, podem determinar desnutrição ou, pelo menos, déficit vitamínico ou mineral ${ }^{6}$.

A maioria dessas perdas funcionais se acentua com a idade devido à insuficiente atividade do sistema neuromuscular, ao desuso e à diminuição do condicionamento físico, determinando complicações e condições debilitantes, inanição, desnutrição, ansiedade, depressão, insônia etc. que, por sua vez, conduzem à imobilidade, desuso, debilidade muscular e enfermidade, estabelecendo-se um círculo vicioso clássico em geriatria. Okuma ${ }^{7}$, comenta que, até recentemente, o treinamento de força para idosos era visto como ineficaz, uma vez que esta diminui com o passar dos anos. Quando estudos com idosos encontravam aumento da força muscular, este era atribuído aos efeitos da aprendizagem do movimento. Os autores consideram que os achados sobre a aparente falta de respostas aos programas de força podem ser explicados mais como uma atitude social negativa dos pesquisadores para com os idosos do que como uma insuficiência de fundamentos teóricos ${ }^{8}$. Há 20 anos, os treinamentos de força para idosos baseavam-se em recomendações de cuidados excessivos e as prescrições de treinamento tendiam, exclusivamente, para trabalhos com carga de baixa intensidade ${ }^{8}$. Muito embora, nos dias atuais esteja aumentando a prática de atividades físicas no âmbito da população idosa, muitos ainda não a realizam e, inclusive, são desaconselhados a participar de programas de condicionamento físico. No que tange às investigações, verifica-se um aumento crescente de pesquisas adequadamente controladas que demonstram resultados significativos do treinamento de força muscular de alta intensidade para idosos, no aumento da força muscular e da hipertrofia muscular, mesmo quando os sujeitos apresentam faixas etárias avançadas ${ }^{7}$.

A prática regular de exercícios físicos é uma estratégia preventiva primária, atrativa e eficaz, para manter e melhorar o estado de saúde física e psíquica em qualquer idade, tendo efeitos benéficos diretos e indiretos para prevenir e retardar as perdas funcionais do envelhecimento, reduzindo o risco de enfermidades e transtornos freqüentes na terceira idade tais como as coronariopatias, a hipertensão, a diabetes, a osteoporose, a desnutrição, a ansiedade, a depressão e a insônia ${ }^{9,5,10}$. Em relação à recuperação da força muscular em idosos, estudos têm demonstrado que ela pode ser conseguida mediante programas de condicionamento físico, de força e resistência, de alta ou baixa intensidade, inclusive em nonagenários ${ }^{6,11}$.

Com essas premissas, o objetivo deste estudo foi verificar a influência de um programa controlado e de longa 
duração (dois anos) sobre a força muscular e a flexibilidade de mulheres idosas.

\section{MÉTODO}

\section{Sujeitos}

Foram sujeitos do presente estudo, 32 indivíduos do sexo feminino, com idade entre 60 e 80 anos, não institucionalizados e residentes na cidade de Salamanca (Espanha). As idosas foram selecionadas dentre o universo de 300 participantes do Programa de Revitalização Geriátrica realizado pela equipe de investigadores da Universidade de Salamanca. Os critérios de seleção foram: ter idade entre 60 e 80 anos, ser do sexo feminino, ter freqüência superior a 74\% nas atividades do programa de atividade física. Todos os participantes assinaram termo de consentimento livre e esclarecido e o programa de revitalização geriátrica foi aprovado pelo comitê acadêmico responsável pelos aspectos éticos, da Universidade de Salamanca.

\section{Material}

Foram utilizados: um manômetro de pressão tipo pêra, com leitura em libras e bares; banco de Wells, com superfície milimetrada e formulários para avaliação e registro de dados.

\section{Procedimento}

Todos os sujeitos foram inicialmente submetidos à avaliação médica que considerava características físicas e históricas de enfermidades pregressas que impedissem a participação em qualquer das atividades previstas no programa. A força muscular foi medida por meio de dinamometria manual dos músculos responsáveis pelo movimento de preensão. Foram tomadas medidas de ambas as mãos, com a realização de três movimentos, sendo que era considerado o maior valor conseguido em cada mão. A flexibilidade corporal foi medida por meio do teste sit and reach (sentar e alcançar), sendo que os indivíduos eram posicionados sentados, com as pernas estendidas e eram orientados a deslocar o marcador sobre uma superfície milimetrada. O movimento era realizado três vezes e considerou-se o maior valor observado.

Os sujeitos realizaram um conjunto de atividades físicas regulares, com 174 sessões, durante 58 semanas, intercaladas com períodos de descanso (ver Figura 1), com freqüência de três vezes por semana e duração de 50 a 55 minutos cada uma. Cada sessão envolveu alongamentos miofasciais passivos dos principais grupos musculares (posteriores da perna e coxa, anteriores da coxa e pelve, vertebrais, paravertebrais, peitorais, etc), com duração de 8 a 10 minutos; ativação cardiocirculatória aeróbia (9 min); exercícios de força, potência e resistência adaptados (7-10 min); atividades de coordenação, agilidade e flexibilidade (14-16 min); exercícios respiratórios e de relaxamento (5-7 min) e hidratação em dois momentos da sessão (25 minutos após o início e ao final da sessão). As medidas foram tomadas antes do início do programa, após 29 semanas de intervenção, após um período intermediário de 18 semanas de descanso e, novamente, após 29 semanas de intervenção (Figura 1).

Os resultados foram analisados por meio do programa estatístico SPSS 11.0, com o teste de ANOVA para medidas repetidas, com o objetivo de identificar diferenças significativas $(p=0,005)$ entre as medidas tomadas nos diferentes períodos.

\section{RESULTADOS}

Em relação à força muscular, na Tabela 1 podem ser visualizadas as médias e os desvios-padrão dos valores encontrados nas quatro medidas realizadas. O que pode ser observado é que os valores médios são distintos nas várias medidas tomadas. É importante destacar que a terceira medida foi tomada após o período de descanso mais longo dos sujeitos (18 semanas). O teste de ANOVA com medidas repetidas não indicou diferenças estatisticamente significativas entre as medidas.

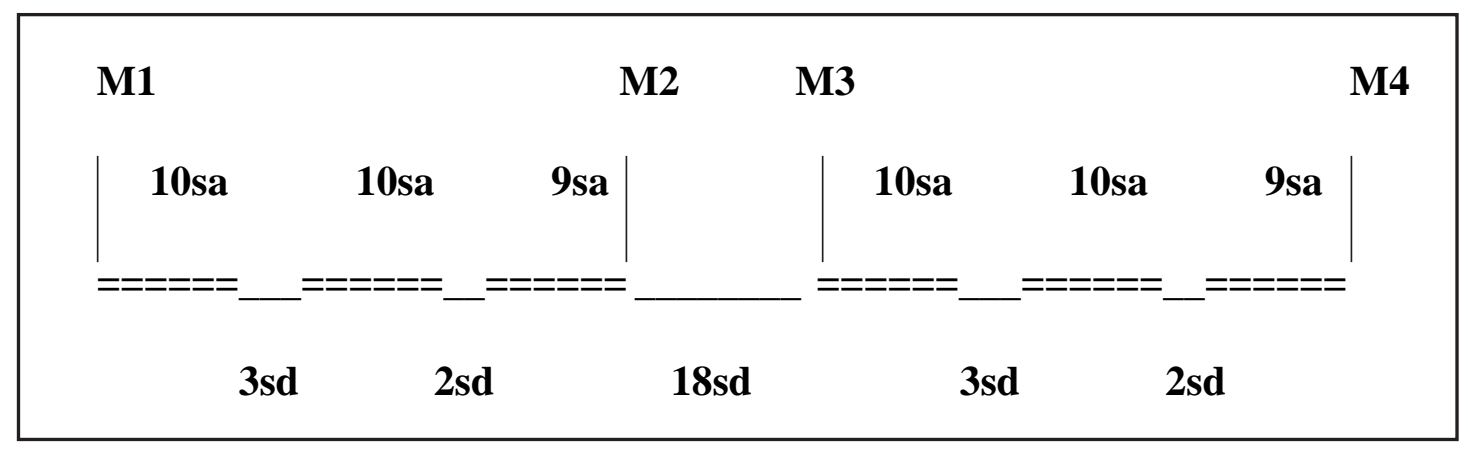

Figura 1. Esquema representativo das tomadas de medida (M), semanas de atividade (sa) e semanas de descanso (sd). 
Tabela 1. Médias e desvios-padrão (dp) dos valores (em Newton/m2) da força de preensão das mãos direita e esquerda, nas quatro medidas tomadas (medida 1 = pré- treinamento; medida 2 = após 29 semanas de treinamento; medida 3 = após descanso e medida 4 = após 58 semanas de treinamento).

\begin{tabular}{lcccc}
\hline & MEDIDA 1 & MEDIDA 2 & MEDIDA 3 & MEDIDA 4 \\
\hline MÃO & 50000 & 53000 & 51000 & 55000 \\
DIREITA & $\mathrm{dp}=10000$ & $\mathrm{dp}=18000$ & $\mathrm{dp}=18000$ & $\mathrm{dp}=18000$ \\
\hline MÃO & 48000 & 50000 & 41000 & 54000 \\
ESQUERDA & $\mathrm{dp}=11000$ & $\mathrm{dp}=19000$ & $\mathrm{dp}=15000$ & $\mathrm{dp}=15000$ \\
\hline
\end{tabular}

Tabela 2. Médias e desvios-padrão (dp) da flexibilidade (em cm), nas quatro medidas tomadas (medida 1 = pré- treinamento; medida 2 = após 29 semanas de treinamento; medida 3 = após descanso e medida 4 = após 58 semanas de treinamento).

\section{MEDIDA 1 MEDIDA 2 MEDIDA 3 MEDIDA 4}

\begin{tabular}{ccccc}
\hline FLEXIBILIDADE & 16,01 & 16,09 & 15,03 & 16,28 \\
\hline$d p=5,6$ & $d p=6,32$ & $d p=5,0$ & $d p=6,28$ \\
\hline
\end{tabular}

Relativamente à flexibilidade muscular, na Tabela 2 estão às medidas obtidas no decorrer do período de desenvolvimento do programa. O que se constata é a ausência de alterações estatisticamente significantes, cabendo destaque também para uma diminuição apenas numérica dos valores na medida tomada após o período de descanso dos sujeitos (medida 3).

\section{DISCUSSÃO}

Inicialmente, o que merece ser comentado é o fato de que as médias da força tanto nas medidas feitas tanto na mão direita como na esquerda apresentaram alterações (Tabela 1) sem significância estatística. Tal aspecto indica que o programa de atividades físicas, mesmo tendo intervalos longos de descanso (um deles com 18 semanas), propiciou a manutenção da força manual bilateral dos sujeitos. Muito embora o programa não tenha tido como objetivo principal o desenvolvimento de músculos específicos, os exercícios realizados com alteres, para o trabalho aeróbio e os apoios sobre o solo parecem ter influenciado nessa manutenção.

Em relação à força muscular, é importante destacar alguns fatores fisiológicos característicos da faixa etária a que pertenciam os sujeitos. Grande parte dos estudos da função neuromuscular no idoso concentra-se na investigação sobre o comportamento da força muscular, que pode ser definida como a capacidade do músculo gerar tensão, e da qualidade muscular, também denominada de tensão específica $^{3}$. Essa última refere-se à força por unidade de massa muscular, podendo constituir-se num melhor indicador de função muscular do que a força isoladamente. O que se verifica é que, normalmente, a força muscular em indivíduos sedentários atinge um pico entre os 20 e 30 anos, tendendo a uma diminuição paulatina nos 20 anos seguintes. A partir dos 65 - 70 anos, a perda de força torna-se mais grave e é 
responsável pelos consideráveis déficits motores observados em indivíduos nessa faixa etária ${ }^{2}$. Harries e Bassey ${ }^{11}$ referemse a um declínio de 15\% entre a sexta e a sétima décadas e, após a sétima década, um declínio de 30\% da força máxima individual a cada década.

Fronteira $^{13}$, em um estudo longitudinal com duração de doze anos, envolvendo homens idosos sedentários (idade inicial em torno de 65 anos) observou declínios de 20 a 30\% da força isocinética máxima dos músculos extensores e flexores do joelho e do cotovelo, durante contrações com velocidades lentas e rápidas.

Considerando essas características fisiológicas e os dados encontrados, é possível afirmar que o programa regular de exercitação física contribuiu para a manutenção da força de preensão de ambas as mãos, evitando os prejuízos decorrentes da perda fisiológica natural da idade. A contribuição verificada pode ser considerada importante para a realização de tarefas de vida diária, se forem levadas em conta, por exemplo, as observações de que os adultos mais velhos são tipicamente capazes de participar de atividades que exigem apenas quantidades moderadas de força muscular. A abertura de um frasco que apresenta resistência é uma tarefa que pode ser facilmente realizada por 92\% dos homens e mulheres na faixa etária de 40 a 60 anos. Porém, após os 60 anos, a taxa de insucesso na realização dessa tarefa aumenta para 68\%. Entre os 71 e 80 anos somente 32\% das pessoas conseguem abrir o frasco ${ }^{14}$.

Segundo Shepard ${ }^{4}$, durante a vida ativa, adultos perdem em torno de 8 a 10 centímetros de flexibilidade na região lombar e no quadril, quando medido por meio do teste de alcance máximo "sit and reach”. Dentre os vários fatores que colaboram para isso estão a maior rigidez de tendões, ligamentos e cápsulas articulares, devido a deficiências no colágeno. Esse mesmo autor cita que a restrição na amplitude do movimento das grandes articulações torna-se mais pronunciada com o envelhecimento e, muitas vezes, a independência funcional é ameaçada porque o indivíduo não consegue utilizar um carro ou um banheiro normal, subir uma escada, ou combinar os movimentos de vestir-se e pentear os cabelos. Aponta ainda que uma das maneiras de conservar a flexibilidade é por meio de movimentos realizados em toda a amplitude das principais articulações.

Os dados que podem ser observados na Tabela 2, da mesma forma com o ocorrido com a força muscular, permitem afirmar que o programa de exercícios evitou que ocorressem as perdas naturais que o processo de senescência determina, considerando que não houve diferenças estatisticamente significativas entre as medidas tomadas no decorrer do período de dois anos. No entanto, como o programa continha exercícios específicos de alongamentos musculares, de realização de movimentos amplos das grandes articulações e mobilização ativa da coluna vertebral, seria natural a expectativa de que as diferenças entre os valores iniciais e finais de flexibilidade fossem mais expressivos, após o referido período. Como isso não ocorreu, tal fato é um indicador para a reprogramação dessas atividades, intensificando-as, de maneira a aumentar a probabilidade de ganhos de flexibilidade corporal. Por outro lado, a manutenção dos valores iniciais é um aspecto que não pode ser desprezado quando se focaliza a qualidade de vida e a independência funcional do indivíduo idoso e a vinculação da força muscular e da flexibilidade corporal com a probabilidade de quedas e de lesões músculoesqueléticas. Em relação a esse fator, Okuma ${ }^{5}$ aponta que para um idoso realizar suas tarefas cotidianas como subir escadas, carregar suas compras e abaixar-se, ele necessita de pouca aptidão cardiovascular, e muito mais de um conjunto de capacidades como força muscular, resistência muscular localizada e flexibilidade, conjunto este denominado de "aptidão muscular”.

\section{CONCLUSÃO}

1.O programa de atividade física regular e de longa duração, mesmo não tendo sido idealizado para o desenvolvimento de força muscular, contribuiu para manutenção da força de preensão bilateral das mãos de mulheres idosas.

2.Embora os dados relativos à flexibilidade corporal indiquem uma possível influência do programa na manutenção de tal capacidade, a ausência de significância estatística indica a necessidade de reprogramação dos exercícios destinados ao ganho da flexibilidade.

\section{REFERÊNCIAS BIBLIOGRÁFICAS}

1. Gonçalves AK. Novo ritmo da terceira idade. Pesquisa Fapesp 2001; 67: p. 68.

2. Duthie EH, Katz PR. Practice of Geriatrics. Philadelphia:Saunders Co; 1998.

3. Fleck SJ, Kraemer WJ. Fundamentos do treinamento de força muscular. Porto Alegre: Artmed; 1999.

4. Shephard RJ. Aging and exercise. Encyclopedia of Sports Medicine and Science. Internet Society for Sport Science 1998.Disponível em: http://sportsci.org.

5. De Jong N, A Paw MJMC, De Groot LCPG ML, De Graaf C, Kok F J, Van Staveren WA. Functional biochemical and nutrient indices in frail erderly people are partly affected by dietary supplements but not by exercise. J Nutr 1999; 129: 2028-36.

6. Fiatarone MA, O'neill EF, Ryan ND, Clements KM, Solares GR, Nelson ME, et al. Exercise training and nutritional supplementation for physical frailty in very elderly people. $\mathrm{N}$ Engl J Med 1994; 330:1769-75.

7. Okuma SS. O idoso e a atividade física: fundamentos e pesquisa. Campinas: Papirus; 2002. 
8. Philips W, Haskell W. Muscular fitness - Easing the burden of desability for elderly adults. Journal of Aging and Physical Activity 1995; 3: 261-289.

9. Lewis RD, Modlesdy CM. Nutrition, physical activity, and bone health in women. Int J Sport Nutr 1998; 8: 250-84.

10. Polidori MC, Mecocci P, Cherubini A. Physical activity and oxidation stress during aging. Int J Sports Med 2000; 21:15457.

11. Charette SL, Mcevoy L, Pyka AG, Snow-Harter C, Guido D, Wiswell A, et al. Muscle hypertrophy response to resistance training in older women. J Appl Physiol 1991; 70: 1912-16.

12. Harries JM, Bassey EJ. Torque- velocity relationship for the knee stensors en women in their 3th na 7rd decades. Eur J Appl Physiol 1990; 60: 87-90.

13. Frontera WR, Hughes VA, Fielding RA, Fiatarone MA, Evans WJ, Roubenoff R. Aging of skeletal muscle: 12-yr longitudinal study. J Appl Physiol 2000; 88: 1321-6.

14. Wilmore JH, Costill DL. Fisiologia do esporte e do exercício. São Paulo:Manole; 2001. 